\title{
Spatiotemporal interference of photoelectron wave packets and the time scale of nonadiabatic transitions in the high-frequency regime
}

\author{
Koudai Toyota (豊田広大)* \\ Center for Free-Electron Laser Science, DESY, 22607 Hamburg, Germany \\ and Max Planck Institute for the Physics of Complex Systems, 01187 Dresden, Germany
}

(Received 28 June 2016; published 11 October 2016)

\begin{abstract}
The method of the envelope Hamiltonian [K. Toyota, U. Saalmann, and J. M. Rost, New J. Phys. 17, 073005 (2015)] is applied to further study a detachment dynamics of a model negative ion in one dimension in the high-frequency regime. This method is based on the Floquet approach, but the time dependency of an envelope function is explicitly kept for arbitrary pulse durations. Therefore, it is capable of describing not only a photon absorption or emission, but also a nonadiabatic transition which is induced by the time-varying envelope of the pulse. It was shown that the envelope Hamiltonian accurately retrieves the results obtained by the time-dependent Schrödinger equation, and the underlying physics were well understood by the adiabatic approximation based on the envelope Hamiltonian. In this paper, we explore two more aspects of the detachment dynamics, which were not considered in our previous work. First, we determine the features of both a spatial and temporal interference of photoelectron wave packets in a photon-absorption process. We conclude that both of the interference mechanisms are universal in ionization dynamics in the high-frequency regime. Second, we extract a pulse duration which maximizes a yield of the nonadiabatic transition as a function of a pulse duration. It is shown that it becomes maximum when the pulse duration is comparable to a time scale of an electron.
\end{abstract}

DOI: 10.1103/PhysRevA.94.043411

\section{INTRODUCTION}

The latest experimental techniques of high-order-harmonic generation can generate coherent light sources in the soft $x$-ray range [1], and have opened up the new realm of the research area called the high-frequency regime. Here the terminology "high frequency" means that a photon energy is high enough to ionize a ground-state electron by single-photon absorption. Meanwhile, the high-frequency regime has been intensively studied in theory for more than three decades in terms of the high-frequency Floquet theory (HFFT) for monochromatic laser fields developed by Gavrila and Kaminski [2]. The HFFT is developed in the Kramers-Henneberger (KH) frame [3]. In the KH frame, the effect of the laser field is described by an atomic potential quivering along a classical trajectory of a free electron in the laser fields. This is called the $\mathrm{KH}$ potential. In the high-frequency limit, where the single optical cycle of the laser field is much shorter than the electron's time scale, it was shown that all of the Fourier components of the $\mathrm{KH}$ potential can be ignored except the zeroth component, i.e., a time average of the $\mathrm{KH}$ potential [2]. This is often called the dressed potential. All of the other photon-absorption or -emission channels then can be treated perturbatively for the bound states of the dressed potential, as these amplitudes are comparable with the dressed potential.

Since the foundation of the HFFT, much of the literature has been involved in the study of ionization dynamics in the high-frequency regime. One of the most striking physical phenomena in the high-frequency regime is stabilization, in which an ionization rate begins to decrease for an intensity higher than a certain critical value, first found by Pont and Gavrila [4]. A great amount of the literature has been devoted to understanding this counterintuitive phenomenon. You et al. [5]

\footnotetext{
*koudai.toyota@cfel.de
}

showed that the stabilization stems from a spatial interference of photoelectron wave packets launched at two turning points of the classical electron in the laser fields.

The HFFT has given us interesting physical insights in the high-frequency regime, but it can only be applied for monochromatic laser fields, i.e., infinite pulse duration. However, laser pulses of the attosecond time scale have become available in the latest experiments, as mentioned above. These unprecedented laser pulses will be employed to study light-matter interactions in an extremely short-time scale in the high-frequency regime, where the effects of a time-varying envelope function of a pulse are expected to play important roles. Therefore, it is highly desirable to develop theoretical methods in the high-frequency regime to adequately treat finite pulse duration beyond the HFFT.

Under such circumstances, we developed the envelope Hamiltonian to treat the photoionization dynamics in the high-frequency regime in our previous work [6]. Photoelectron amplitudes were analytically derived in the framework of the adiabatic approximation based on the envelope Hamiltonian. The procedures follow the HFFT, i.e., we realize a dressed potential and treat photon-absorption or -emission channels perturbatively, but the time dependency of a pulse envelope explicitly remains. Thus we also obtain the photoelectron amplitudes for a nonadiabatic transition induced by the timedependent envelope function which does not show up in the original HFFT. The capability of the envelope Hamiltonian and the adiabatic approximation was demonstrated in [6] utilizing a simple model in one dimension in the stabilization regime. It was shown that the results obtained by the full time-dependent Schrödinger equation (TDSE) calculations were accurately reconstructed by the TDSE for the envelope Hamiltonian.

In this paper, we further explore the ionization dynamics in the high-frequency regime working on two subjects utilizing the envelope Hamiltonian. First, we revisit the oscillating substructure in photon-absorption peaks in the high-frequency 
regime, which has been recently studied by several groups [7-10]. In [7], they found the oscillating structure in the stabilization regime, and reconstructed it taking into account a spatial and temporal interference of photoelectron wave packets. However, their formulas were obtained in an empirical way, bringing a quasistatic picture into the HFFT. On the other hand, in [8-10], they only addressed the temporal interference although their theoretical approach was based on first principles. In this paper, we find the signature of both the spatial and temporal interference in the formula obtained from first principles. The temporal interference in photo electron spectra has been also found in the low-frequency regime; see [11] and [12], for instance.

The second subject of this paper is to extract an optimal pulse duration to maximize a yield of the nonadiabatic transition. It was found in our previous work that the yield as a function of a pulse duration has a maximum at a certain pulse duration [6]. We find a formula to predict the peak position and show that the yield becomes maximum when the pulse duration is close to a time scale of an electron. These two subjects have not been explored extensively in the high-frequency regime due to the lack of appropriate theoretical frameworks which can take into account time-varying envelope functions. We strongly believe that these subjects are worth studying to gain further insights into the ionization dynamics in the high-frequency regime.

This paper is thus organized as follows. In Sec. II, we introduce our theoretical methods. We briefly summarize the formulations in [6] for a case of one dimension to refer to them later. In Secs. II A-II C, we derive the envelope Hamiltonian in the $\mathrm{KH}$ frame employing a normalized classical trajectory. In Sec. II D, we derive photoelectron amplitudes for the photonabsorption or -emission processes and nonadiabatic transition. After giving short remarks on the nonadiabatic transition in Sec. II E, we extract the evidence of the spatial and temporal interference in the formula of the photon-absorption spectrum with the aid of the saddle-point method in Sec. II F. In Sec. III, we study a detachment dynamics of a model negative ion in the high-frequency regime to demonstrate our theory. In Sec. III A, we revisit the oscillating structure in photon-absorption peaks to confirm that our theory is consistent with previously known results [7-10]. In Sec. III B, we find an optimal pulse duration to maximize a yield of the nonadiabatic transition. In Sec. IV, we conclude the paper with future perspectives. Atomic units are used throughout the paper.

\section{THEORETICAL METHODS}

In this section, we summarize our theoretical method [6] in one dimension to refer to it later.

\section{A. TDSE in the Kramers-Henneberger frame} reads

The time-dependent Schrödinger equation in one dimension

$$
H(t)|\Psi(t)\rangle=i \frac{\partial}{\partial t}|\Psi(t)\rangle .
$$

The Hamiltonian $H(t)$ within the dipole approximation in the Kramers-Henneberger $(\mathrm{KH})$ frame is given by

$$
H(t)=-\frac{1}{2} \frac{\partial^{2}}{\partial x^{2}}+V\left(x+x_{\omega}(t)\right)
$$

where $V(x)$ is an atomic potential. The function $x_{\omega}(t)$ represents a classical trajectory of a free electron in a laser pulse $F(t)$,

$$
\frac{d^{2} x_{\omega}}{d t^{2}}=-F(t)
$$

The laser pulse $F(t)$ satisfies

$$
\begin{aligned}
\lim _{t \rightarrow \pm \infty} F(t) & =0 \\
\int_{-\infty}^{\infty} F\left(t^{\prime}\right) d t^{\prime} & =0 .
\end{aligned}
$$

In the $\mathrm{KH}$ frame, external fields are described by the quivering motions of the atomic potential along the classical trajectory given by Eq. (3), and this potential is called the $\mathrm{KH}$ potential.

\section{B. Normalized classical trajectory}

We define the classical trajectory $x_{\omega}(t)$ in Eq. (2) as

$$
x_{\omega}(t)=\alpha(t) \cos (\omega t+\delta) .
$$

Then the pulse is given by Eq. (3). The function $\alpha(t)$ is the envelope of the pulse given by

$$
\alpha(t)=\alpha_{0} e^{-a\left(\frac{t}{T}\right)^{2}},
$$

where $a=2 \ln (2)$ so that a pulse duration $T$ is defined by the full width at half maximum (FWHM) of $F^{2}(t)$. The constant $\alpha_{0}$ is given by

$$
\alpha_{0}=\frac{F_{0}}{\omega^{2}+\frac{2 a}{T^{2}}}
$$

so that the peak field amplitude becomes $F(0)=F_{0} \cos \delta$. Note that our pulse $F(t)$ defined in this way satisfies the conditions given by Eqs. (4). In this paper, we consider the values of a photon energy $\omega$ much higher than an ionization potential of a ground state $I_{p}$,

$$
\omega \gg I_{p}
$$

\section{The envelope Hamiltonian}

In [6], it was shown that the full Hamiltonian given by Eq. (2) in the high-frequency regime, given by Eq. (8), is accurately approximated by the envelope Hamiltonian,

$$
H_{\mathrm{env}}(t)=H_{0}(t)+U(x, t),
$$

where $H_{0}(t)$ and $U(x, t)$ are given by

$$
\begin{aligned}
H_{0}(t) & =-\frac{1}{2} \frac{\partial^{2}}{\partial x^{2}}+V_{0}(x, t), \\
U(x, t) & =\sum_{n= \pm 1, \pm 2} V_{n}(x, t) e^{-i n \omega t} .
\end{aligned}
$$

The function $V_{n}(x, t)$ is defined by

$$
V_{n}(x, t)=\frac{1}{T_{\omega}} \int_{0}^{T_{\omega}} V\left(x+\alpha(t) \cos \left(\omega t^{\prime}+\delta\right)\right) e^{i n \omega t^{\prime}} d t^{\prime},
$$

where the quantity $T_{\omega}=2 \pi / \omega$ represents the single optical cycle. This function represents one of the components in the 
momentary Fourier expansion of the KH potential in (2) for a given value of the envelope function $\alpha(t)$,

$$
V\left(x+x_{\omega}(t)\right)=\sum_{n=-\infty}^{\infty} V_{n}(x, t) e^{-i n \omega t} .
$$

The function $V_{0}(x, t)$ reduces to the atomic potential $V(x)$ for $t \rightarrow \pm \infty$. So, the quantity $H_{0}(t)$, given by Eq. (10a), represents a distorted Hamiltonian of the electron in the action of the pulse. It is often called the dressed Hamiltonian.

The functions for $n \neq 0$ represent $|n|$ photon-absorption or -emission channels, depending on whether the sign is positive or negative. It must be realized that we only need the $n=0, \pm 1$, and \pm 2 terms to approximate the full Fourier expansion given by Eq. (10d) in the high-frequency regime [6]. This is valid not only for a long pulse limit, but also a short pulse limit. In the long pulse limit, multiphoton-absorption or -emission channels dominate ionization dynamics. Since the matrix elements for them involve high-energy scattering states, which are highly oscillatory in space, these amplitudes are very small. So, we only retain up to $|n|=2$. For the short pulse limit, it is found that the quiver amplitude $\alpha_{0}$ given by Eq. (7) is very small. Considering the Taylor expansion of the functions $V_{n}(x, t)$ for $n=0, \pm 1$, and \pm 2 , and summing them according to the manner of Eq. (10d), the result coincides with the full $\mathrm{KH}$ potential with $O\left(\alpha^{2}(t)\right)$.

We consider the TDSE for $H_{\mathrm{env}}(t)$,

$$
H_{\mathrm{env}}(t)|\psi(t)\rangle=i \frac{\partial}{\partial t}|\psi(t)\rangle .
$$

We call this equation the envelope TDSE. The equivalent Hamiltonian for the case of monochromatic fields was also considered by Henneberger in [3].

\section{Adiabatic approximation for photoionization}

In this section, we derive the photoelectron amplitudes in one dimension based on the envelope Hamiltonian, given by Eq. (9), to utilize them in later sections. The full derivations with an arbitrary number of bound states in three dimensions are found in [6]. Let $\left|\phi_{0}(t)\right\rangle$ and $|k, t\rangle$ be a ground state and scattering state of the dressed Hamiltonian $H_{0}(t)$ given by Eq. (10a),

$$
\begin{aligned}
H_{0}(t)\left|\phi_{0}(t)\right\rangle & =E_{0}(t)\left|\phi_{0}(t)\right\rangle, \\
H_{0}(t)|k, t\rangle & =E|k, t\rangle,
\end{aligned}
$$

where

$$
E=\frac{k^{2}}{2}
$$

The orthogonality is

$$
\begin{aligned}
\left\langle\phi_{0}(t) \mid \phi_{0}(t)\right\rangle & =1, \\
\left\langle k, t \mid k^{\prime}, t\right\rangle & =2 \pi \delta\left(k-k^{\prime}\right) .
\end{aligned}
$$

Employing them, we expand the solution of the envelope TDSE given by Eq. (11),

$$
\begin{aligned}
|\psi(t)\rangle= & e^{-i \chi(t)}\left[C_{0}(t)\left|\phi_{0}(t)\right\rangle e^{-i \int_{-\infty}^{t} E_{0}\left(t^{\prime}\right) d t^{\prime}}\right. \\
& \left.+\int_{-\infty}^{\infty} C_{k^{\prime}}(t)\left|k^{\prime}, t\right\rangle e^{-i E^{\prime} t} \frac{d k^{\prime}}{2 \pi}\right]
\end{aligned}
$$

where $E^{\prime}=k^{\prime 2} / 2$ and the coefficients $C_{0}(t)$ and $C_{k}(t)$ represent the ground-state population and photoelectron amplitude of momentum $k$ for a certain time $t$, respectively. The phase $\chi(t)$ is given by

$$
\chi(t)=\int_{-\infty}^{t}\left\langle\phi_{0}\left(t^{\prime}\right)\left|U\left(x, t^{\prime}\right)-i \frac{\partial}{\partial t^{\prime}}\right| \phi_{0}\left(t^{\prime}\right)\right\rangle d t^{\prime},
$$

so that coupled differential equations for the coefficients $C_{0}(t)$ and $C_{k}(t)$ become simple. Let us introduce the notation

$$
\Phi_{n}(t)=-\int_{-\infty}^{t} E_{0}\left(t^{\prime}\right) d t^{\prime}-n \omega t+E t .
$$

Substituting the expansion Eq. (15) into the envelope TDSE given by Eq. (11), we obtain the coupled equations for the coefficients $C_{0}(t)$ and $C_{k}(t)$. The coupled equations can be solved perturbatively. Let us assume the zeroth-order solution as $C_{0}^{(0)}(t)=1$ and $C_{k}^{(0)}(t)=0$. Then the first-order solution is given by

$$
\begin{aligned}
& C_{0}^{(1)}(t)=1, \\
& C_{k}^{(1)}(t)=-i \int_{-\infty}^{t} Q_{k 0}\left(t^{\prime}\right) e^{i \Phi_{0}\left(t^{\prime}\right)} d t^{\prime} .
\end{aligned}
$$

The quantity $Q_{k 0}(t)$ is given by

$$
Q_{k 0}(t)=\left\langle k, t\left|U(x, t)+i \frac{\dot{V}_{0}(x, t)}{E-E_{0}(t)}\right| \phi_{0}(t)\right\rangle,
$$

where $\dot{V}_{0}$ is the time derivative of $V_{0}$. The photoelectron spectrum $d p / d k$ is thus approximated as

$$
\frac{d p}{d k} \simeq\left|\lim _{t \rightarrow \infty} C_{k}^{(1)}(t)\right|^{2}=\left|\sum_{n=-2}^{2} C_{n \omega}^{(1)}(k)\right|^{2} .
$$

The function $C_{n \omega}^{(1)}(k)$ is defined by

$$
C_{n \omega}^{(1)}(k)=\int_{-\infty}^{\infty} M_{n \omega}(k, t) e^{i \Phi_{n}(t)} d t,
$$

where

$$
M_{n \omega}(k, t)= \begin{cases}\left\langle k, t\left|i \frac{\dot{V}_{0}(x, t)}{E-E_{0}(t)}\right| \phi_{0}(t)\right\rangle & (n=0), \\ \left\langle k, t\left|V_{n}(x, t)\right| \phi_{0}(t)\right\rangle & (n \neq 0) .\end{cases}
$$

\section{E. Nonadiabatic transition}

The total photoelectron amplitude given by Eq. (21a) consists of two different kinds of physical processes. The first one is given by $n=0$ in Eq. (21b), which represents the nonadiabatic transitions to the continuum induced by the time dependency of the ground state $\left|\phi_{0}(t)\right\rangle$. Therefore, one speculates that the following TDSE is responsible for the nonadiabatic transition:

$$
H_{0}(t)\left|\psi_{0}(t)\right\rangle=i \frac{\partial}{\partial t}\left|\psi_{0}(t)\right\rangle .
$$

The TDSE given by Eq. (22) accurately approximates the full TDSE given by Eq. (1) if the contributions of multiphoton absorption or emission to the total ionization yield are negligibly small. The TDSE given by Eq. (22) was realized in [13] to study the nonadiabatic transition between bound 
states in one dimension. In the paper, they predicted that an electron is ionized with low energy. In Eq. (21b) for $n=0$, the integrand has a large amplitude around the energy $E=E_{0}(t)<0$, and tends to be zero as $E \rightarrow \infty$. So it is expected that the nonadiabatic transition ionizes an electron with low energies. The generation of a slow electron in the high-frequency regime was confirmed in [14]. In the paper, it was found when the electron is subjected to a square-shaped pulse, i.e., a sudden jump between a field-free and dressed ground state is responsible for the emergence. However, the mechanism is different from the nonadiabatic transition here since the time derivative of the dressed potential $V_{0}(x, t)$ in Eq. (10a) cannot be defined at the moment of the sudden ramp of the pulse. Later, the emergence of the slow electron in the context of the nonadiabatic transition was obtained in [15], whose spectrum for a long pulse limit was studied in terms of the adiabatic approximations to the transitions to the continuum [16]. More recently, the slow electron was also found in the study of an above-threshold ionization spectrum of a carbon atom in a hard x-ray regime [17]. They explained its emergence by the Raman-type process, i.e., a single-photon absorption followed by a single-photon emission.

\section{F. Spatial and temporal interference of photoelectron wave packets}

Another contribution in Eq. (21a) is defined by Eq. (21b) with $n \neq 0$. For positive (negative) values of $n$, the quantity represents the photoelectron amplitude by $|n|$ photon absorption (emission). The stationary phase condition is given by

$$
E=E_{0}(t)+n \omega .
$$

This equation has two solutions, $t=t_{\mp}\left(t_{+}=-t_{-}\right)$, in the rising and falling part of the pulse, respectively. Taking into account these two solutions, the photon-absorption spectrum is approximated to

$$
\left|C_{n \omega}^{(1)}(k)\right|^{2} \approx \frac{4 \Gamma_{n}\left(t_{+}\right)}{\left|\dot{E}_{0}\left(t_{+}\right)\right|} \cos ^{2}\left[\theta\left(t_{+}\right)+\frac{\pi}{4}\right],
$$

where

$$
\begin{aligned}
\Gamma_{n}\left(t_{+}\right) & =2 \pi\left|M_{n \omega}\left(k, t_{+}\right)\right|^{2}, \\
k(t) & = \pm \sqrt{2\left[E_{0}(t)+n \omega\right]} .
\end{aligned}
$$

The function $\Gamma_{n}(t)$ represents the $n$ photoionization rate at a given time $t$, and the quantity $k$ represents the momentum of the ionized electron. The sign of $k$ corresponds to an electron ionizing to the right $(+)$ and left $(-)$ direction, respectively. In the derivation of Eq. (24a), the relations of $\Gamma_{n}\left(t_{-}\right)=\Gamma_{n}\left(t_{+}\right)$ and $\dot{E}_{0}\left(t_{-}\right)=\left|\dot{E}_{0}\left(t_{+}\right)\right|$are used; our envelope function $\alpha(t)$, given by Eq. (6), is symmetric with respect to $t=0$. The function $\theta\left(t_{+}\right)$is given by

$$
\theta\left(t_{+}\right)=-\int_{0}^{t_{+}} E_{0}(t) d t+E_{0}\left(t_{+}\right) t_{+}
$$

It is found in Eq. (24a) that two different interference mechanisms contribute to the spectrum.

The first mechanism is a spatial interference found in the $n$ photoionization rate $\Gamma_{n}(t)$. In Fig. 1(a), the broken (red) line shows the single-photon-absorption rate as a function of a time, given by Eq. (24b) with $n=1$, on the right axis for a model potential of $\mathrm{H}^{-}$[Eq. (29)]. The peak field amplitude, photon energy, and pulse duration are $F_{0}=0.5, \omega=\pi / 10$, and $T=2000$, respectively. The amplitude of the envelope function $\alpha(t)$, given by Eq. (6), is shown by the solid (black) line on the left axis as a reference. We see that the rate increases in the beginning part of the pulse as the amplitude of the envelope grows. However, it starts decreasing around the time $t=-0.8 T$ even though the envelope function is still growing. This is the stabilization [4] which is the evidence of the spatial interference of two photoelectron wave packets in a destructive way [5]. This counterintuitive phenomenon is well understood in terms of the dressed potential $V_{0}(x, t)$ in Eq. (10a). By the definition given by Eq. (10c) with $n=0$, our dressed potential is obtained by the time average of the $\mathrm{KH}$ potential, which quivers along the classical trajectory given by Eq. (5), over a single optical cycle for a given value of the envelope function at a time $t$. The velocity of the quivering motion of the $\mathrm{KH}$ potential is the maximum when it passes through the origin and zero at the turning points of the classical trajectory at $x= \pm \alpha(t)$. Therefore, in the dressed potential, the probability of finding the electron is high around these turning points. The photoelectron wave packets are thus launched from the two turning points and they interfere in the picture of the KH frame. The shape of the dressed potential before the occurrence of the stabilization $(t=-1.5 T)$ is shown by the broken line in Fig. 1(b). Because the quiver amplitude is only $\alpha(t=-1.5 T) \approx 0.22$, two photoelectron wave packets are produced from almost the same position in space. Therefore, they constructively interfere. However, the distance of the two turning points becomes larger as the amplitude of the envelope $\alpha(t)$ grows, and the picture of interference turns into destructive at a certain moment in time. The moment in this example is given by $t \approx-0.8 T$. Due to the destructive interference, the net amplitude of the photoelectron wave packet is suppressed, and this emerges as the decrease of the rate, as shown in Fig. 1(a). This is the stabilization. The shape of the dressed potential after the emergence of the stabilization $(t=0)$ is shown by the solid line in Fig. 1(b). The double-well structure reflects the turning points $x= \pm \alpha(t)$ and more clearly shows us that two photoelectron wave packets are created around these points. The double-well structure of the dressed potential in the stabilization regime is often called dichotomy; see [18] for instance.

The phase difference of these two photoelectron wave packets for the spatial interference at a moment $t$ is expected to be the function of the momentary distance between the two turning points $2 \alpha(t)$. This is indeed the case. Employing the derivation by Pont [19], regarding the envelope function $\alpha(t)$ as an adiabatic variable, the rate $\Gamma_{n}(t)$ in the high-frequency limit $\omega \rightarrow \infty$ can be approximated as

$$
\Gamma_{n}(t) \approx|A(k(t))|^{2}\left|\varphi_{0}(\alpha(t))\right|^{2} J_{n}^{2}(|k(t)| \alpha(t)),
$$

where

$$
A(k(t))=\int_{-\infty}^{\infty} V(x) e^{i k(t) x} d x
$$

and

$$
\varphi_{0}(-\alpha(t))=\frac{1}{T_{\omega}} \int_{0}^{T_{\omega}} \phi_{0}\left(-\alpha(t) \cos \left(\omega t^{\prime}+\delta\right)\right) d t^{\prime}
$$



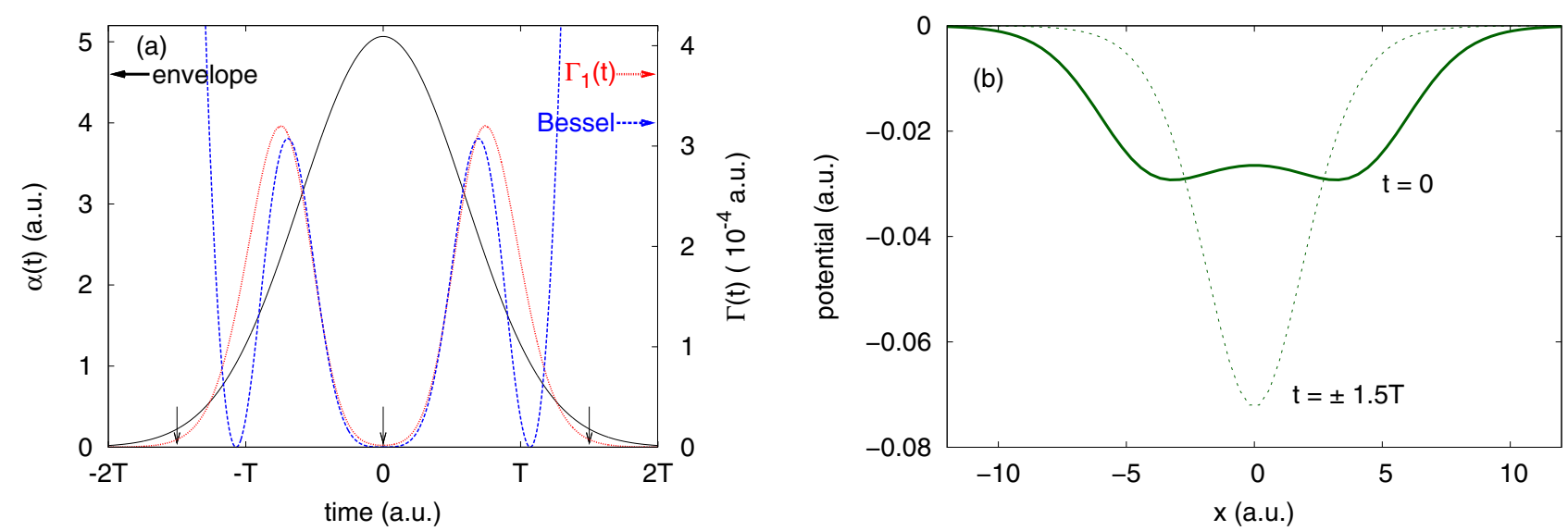

FIG. 1. (a) The comparison of the single-photon-absorption rate, given by Eq. (24b) with $n=1$, as a function of time, shown by the broken (red) line, with its asymptotic form, given by Eq. (28), shown by the dashed (blue) line, on the right axis. The envelope function with Eq. (6) as a reference is shown on the left axis. (b) The dressed potentials at $t= \pm 1.5 T$ and 0 , which are indicated by the vertical arrows in (a).

and the function $J_{n}(z)$ represents the Bessel function of the $n$th order. In the derivation of Eq. (25a), the scattering state $|k, t\rangle$ is approximated as

$$
|k, t\rangle \approx e^{i k(t) x}
$$

and also the following function as

$$
\begin{aligned}
\phi_{0}\left(-\alpha(t) \cos \left(\omega t^{\prime}+\delta\right)\right) & =\sum_{m=-\infty}^{\infty} \varphi_{m}(-\alpha(t)) e^{-i m \omega t^{\prime}} \\
& \approx \varphi_{0}(-\alpha(t)) .
\end{aligned}
$$

We would emphasize that the functional form of Eq. (25a) is universal, i.e., it does not depend on dimensionality and the number of bound states in an atomic potential. Indeed, Pont's derivation was done for a hydrogen atom in three dimensions [19]. Applying the asymptotic expansion for the Bessel function $J_{n}(z)$ for large arguments $z \gg 1$, Eq. (25a) for $n=1$ reduces to

$$
\begin{aligned}
\Gamma_{1}(t) \sim & \frac{1}{\pi|k(t)| \alpha(t)}|A(k(t))|^{2}\left|\varphi_{0}(\alpha(t))\right|^{2} \\
& \times\{1-\sin [2|k(t)| \alpha(t)]\} .
\end{aligned}
$$

In Fig. 1(a), the result of Eq. (28) labeled "Bessel" is shown by the dashed (blue) line. Although the result diverges for small values of $\alpha(t)$ due to the factor of $1 / \alpha(t)$, the behavior of the single photoionization rate around the stabilization is very well reproduced. Hence we understand that the two photoelectron wave packets produced at two turning points $x= \pm \alpha(t)$ interfere with the phase difference, which is the function of $2|k(t)| \alpha(t)$.

The second interference mechanism in Eq. (24a) is the temporal interference imprinted in the cos function. In the falling part of the pulse, the distance between the two turning points shrinks as the amplitude of the envelope function decreases. Then the rate starts increasing until $T \approx 0.8 T$, and decreases again after that. Overall, the rate shows clearly separated peaks before and after the peak intensity. This tells us that the creation of a photoelectron wave packet by the spatial interference takes place twice, i.e., in the rising and falling part of the pulse, and they interfere with the phase difference which stems from the different moment of their birth in time. This causes the temporal interference. The formula quite similar to Eq. (24) was found in the study of the oscillating substructure in photon-absorption peaks in [7]. The difference is that our formula does not take into account the depletion of the ground state since Eq. (24a) is obtained from the first-order solution given by Eqs. (18). However, the formula in [7] was obtained in an empirical manner introducing a quasistatic picture into the HFFT. Here, we obtain the formula given by Eq. (24a) from first principles, which can capture the signatures of both the spatial and temporal interference in the spectrum. After the findings in [7], several groups also found the temporal interference in the high-frequency regime, for a hydrogen atom in [8] and also for a hydrogen molecular ion [9,10]. However, in these studies, only the temporal interference was discussed.

\section{G. Numerical implementations}

In this paper, we mainly study a photodetachment of the hydrogen negative ion $\mathrm{H}^{-}$in one dimension with single active electron approximation. The electron's potential is modeled by [20]

$$
\begin{aligned}
V(x) & =-D \frac{\exp \left[-\sqrt{x^{2}+a^{2}}\right]}{\sqrt{x^{2}+b^{2}}}, \\
D & =24.856, a=4, b=6.27 .
\end{aligned}
$$

This potential supports only one bound state, $E_{0}=$ -0.0277 . We employ the Siegert state expansion method in the $\mathrm{KH}$ frame, previously developed in [7] to solve the full TDSE given by Eq. (1), the envelope TDSE given by Eq. (11), and also Eq. (22).

\section{RESULTS}

\section{A. Revisit of the oscillating substructure in photon-absorption peaks}

Figure 2 shows a photoelectron spectrum near a position of a photo peak $E=E_{0}( \pm \infty)+\omega$ for a set of laser parameters $F_{0}=0.5, \omega=\pi / 10$, and $T=2000$. The solid line (red) and 


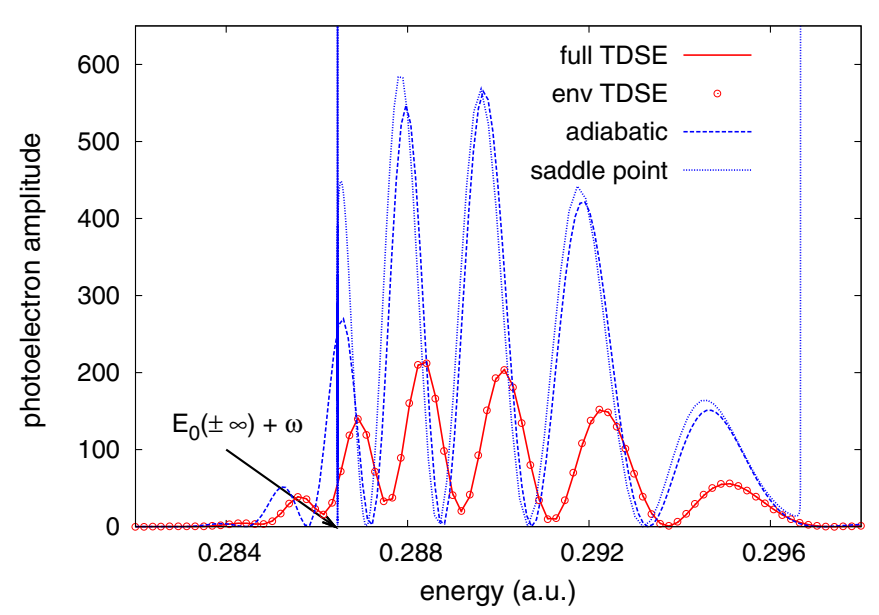

FIG. 2. The photoelectron spectrum for the laser parameters $F_{0}=$ $0.5, \omega=\pi / 10$, and $T=2000$ near a photo peak $E=E_{0}( \pm \infty)+\omega$ indicated by an arrow. The solid (red) line and blank (red) circles are obtained by the full TDSE given by Eq. (1) and envelope TDSE given by Eq. (11), respectively. The broken (blue) and dashed (blue) lines are obtained from the adiabatic approximation given by Eq. (21a) with $n=1$ and the saddle-point method given by Eq. (24a), respectively.

blank circles (red) represent the result of the full TDSE given by Eq. (1) and envelope TDSE given by Eq. (11), respectively. It is clearly shown that the envelope TDSE perfectly reproduces the full TDSE result. The overall structure is blueshifted with respect to the position of the photo peak at $E=E_{0}( \pm \infty)+\omega$ since the ground-state energy $E_{0}(t)$ becomes shallower during the action of the pulse. The broken (blue) and dashed (blue) lines represent the result of the adiabatic approximation given by Eq. (21a) for $n=1$ and the saddle-point method given by Eq. (24a). Their amplitudes are quite overestimated compared to the full TDSE calculation because the depletion of the ground state is ignored in the adiabatic approximation. And the phase shift of the interference structure is found for these results with respect to the full TDSE result given by Eq. (1). This is also due to ignoring the depletion in the adiabatic approximation; the photoelectron amplitude produced in the falling part of the pulse is largely overestimated. So, the relative phase of it with respect to that produced in the rising part of the pulse deviates from the exact calculation. The divergence around $E \approx E_{0}( \pm \infty)+\omega=0.2864$ and $E \approx E_{0}(0)+\omega=0.2965$ in the saddle-point method is seen because the time derivative of the ground-state energy vanishes; see Eq. (24a). The former comes from the rising and falling edge of the pulse where the Stark dressing to the ground state is very small, and the latter at the peak intensity of the pulse. Since the saddle points $t_{-}$and $t_{+}$coalesce at the peak intensity, the divergences around the high-energy edge can be removed by the uniform approximation [21]. The procedure is given in Appendix C. We consider that the oscillating substructure can be understood very well with the adiabatic approximation and saddle-point method. Therefore, we thus conclude that the oscillating structure in the photon-absorption peak in the high-frequency regime is formed by the spatial and temporal interference of photoelectron wave packets discussed in Sec. II F.

\section{B. Time scale of nonadiabatic transition}

In this section, we solve Eq. (22) for several model potentials to study the nonadiabatic transition. In the previous work [6], it was found that a yield of the nonadiabatic transition has a maximum as a function of a pulse duration. One may consider that this is a mathematical artifact due to the normalization factor $\alpha_{0}$, given by Eq. (7), for our classical trajectory given by Eq. (5). For a limit of a pulse duration $T \rightarrow 0$, the yield vanishes because $\alpha_{0}$ becomes zero. Note that the time derivative of $V_{0}(x, t)$ in Eq. (21b) is of the order of $\alpha_{0}^{2}$ in this limit. And the yield also vanishes for a limit of $T \rightarrow \infty$ because the dressed potential $V_{0}(x, t)$ varies infinitely slowly in time. Therefore, it is no wonder that a maximum can be found in between. The quantity $\alpha_{0}$ as a function of $T$ starts from the origin and rapidly converges to its asymptotic value $F_{0} / \omega^{2}$ as $T \rightarrow \infty$. The example is shown by the broken (green) line in Fig. 3 on the right axis. If the maximum yield of the nonadiabatic transition is found in the region where the quantity $\alpha_{0}$ is rapidly growing, the occurrence is concluded to be just a mathematical artifact. After the maximum, the yield of the nonadiabatic transition then starts to decrease because the dressed potential does not significantly vary anymore in time; the electron enters into the adiabatic regime. So, the yield does not increase even the value of $\alpha_{0}$ is still growing. Therefore, the maximum is formed by the fact that the yield of the nonadiabatic transition is proportionate to $\alpha_{0}^{2}$ for small values of $T$. However, the maximum shown in the following takes place far away from the region. Hence, the maximum has a physical origin.

We consider small values of $\alpha_{0}$ to facilitate ourselves to analytically derive formulas to extract the physics of the nonadiabatic transition. Up to the second order of $\alpha^{2}(t)$, a photoelectron amplitude for the nonadiabatic transition $C_{0 \omega}^{(1)}(k)$, given by Eq. (21a) for $n=0$, is reduced to

$$
\begin{aligned}
C_{0 \omega}^{(1)}(k) & \approx D(k) f(E), \\
D(k) & =\left\langle k, t=-\infty\left|V^{\prime \prime}(x)\right| \phi_{0}(-\infty)\right\rangle, \\
f(E) & =-\frac{a}{T^{2}} \frac{1}{E-E_{0}( \pm \infty)} \int_{-\infty}^{\infty} t \alpha^{2}(t) e^{i \Phi_{0}(t)} d t .
\end{aligned}
$$

The function $\Phi_{0}(t)$ is given in Eq. (17). For simplicity, we ignore the Stark shift in the function $f(E)$. Then the function $f(E)$ is reduced to

$$
f(E) \approx-i \sqrt{\frac{\pi}{32 a}} \alpha_{0}^{2} T e^{-\frac{T^{2}}{8 a}\left[E-E_{0}( \pm \infty)\right]^{2}} .
$$

Therefore, we obtain the approximated spectrum of the nonadiabatic transition,

$$
\left|C_{0 \omega}^{(1)}(k)\right|^{2}=\frac{\pi}{32 a}|D(k)|^{2} \alpha_{0}^{4} T^{2} e^{-\frac{T^{2}}{4 a}\left[E-E_{0}( \pm \infty)\right]^{2}} .
$$

Next, we derive the formula for the detachment yield. To implement this, we consider an ansatz for the functional form of $|D(k)|^{2}$,

$$
|D(k)|^{2}=c_{1} k^{2} e^{-c_{2} \frac{k^{2}}{4 a I_{p}}},
$$

where $I_{p}=\left|E_{0}( \pm \infty)\right|$. The detachment probability $P_{0}(T)$ as a function of a pulse duration $T$ is calculated substituting 

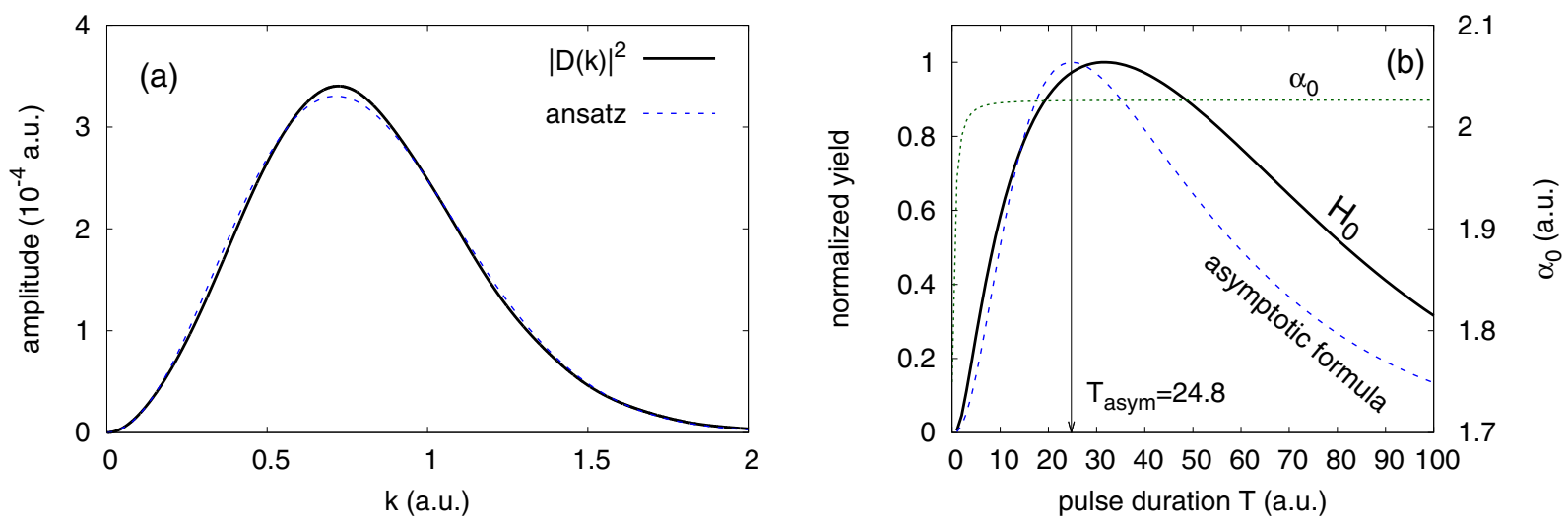

FIG. 3. (a) Comparison between the numerically calculated matrix element $D(k)$ [Eq. (30b)] and its fitting by the ansatz [Eq. (33)]. (b) The solid (black) line shows the detachment yield by the nonadiabatic transition for our model [Eq. (29)] as a function of pulse duration $T$ obtained solving Eq. (22) in the high-frequency limit $\omega \rightarrow \infty$. It is enough to consider $m=8$ in Eq. (35), i.e., $\omega=4 \pi / 5$ and $F_{0}=12.8$, to reach the limit. The dashed (blue) line is obtained using the asymptotic formula [Eq. (37)]. These are shown on the left axis. The heights of these curves are normalized to unity to clearly compare the positions of the peaks. The broken (green) line shows the normalization factor $\alpha_{0}$ of our envelope function [Eq. (7)] on the right axis.

Eq. (33) into (32), and integrating over $k$,

$$
\begin{aligned}
P_{0}(T) & =\int_{-\infty}^{\infty}\left|C_{0 \omega}^{(0)}(k)\right|^{2} \frac{d k}{2 \pi} \\
& =\frac{c_{1}}{2^{\frac{15}{4}} a^{\frac{1}{4}}} \alpha_{0}^{4} \sqrt{T} \xi^{\frac{3}{2}} e^{\xi^{2}-\frac{\left(I_{p} T\right)^{2}}{4 a}}\left[K_{\frac{3}{4}}\left(\xi^{2}\right)-K_{\frac{1}{4}}\left(\xi^{2}\right)\right],
\end{aligned}
$$

where $\xi$ is defined as

$$
\xi=\frac{1}{\sqrt{8 a}} \frac{\left(I_{p} T\right)^{2}+c_{2}}{I_{p} T} .
$$

The derivation is found in Appendix A. In Appendix B, it is clarified that the constant $c_{2}$ is related to the curvature of the atomic potential. Before proceeding with further derivations, we consider the high-frequency limit $\omega \rightarrow \infty$. This consideration facilitates us with the following. First, we can make the function $\alpha_{0}$ as a function of $T$ more rapidly converge to its asymptotic value $F_{0} / \omega^{2}$ as $T$ grows. This makes it easy to more clearly separate the region of this rapid growth and the occurrence of the maximum of the nonadiabatic transition. Second, the dependency on $T$ of the function $\alpha_{0}$ is weakened around the values of $T$ where the maximum yield of the nonadiabatic transition takes place. Below, we do not consider the dependency on $T$ of the function $\alpha_{0}$ when we calculate $d P_{0} / d T$. Finally, the contribution to the total ionization yield by the photon absorption or emission, given by Eq. (21b) with $n \neq 0$, vanishes in the high-frequency limit. So, we can safely replace the full TDSE given by Eq. (1) with Eq. (22). To numerically arrive at the high-frequency limit $\omega \rightarrow \infty$, we introduce a scaling of $F_{0}$ and $\omega$ as

$$
F_{0} \rightarrow m^{2} F_{0} \text { and } \omega \rightarrow m \omega,
$$

to keep a ratio $F_{0} / \omega^{2}$ being a constant, where $F_{0}=0.2$ and $\omega=\pi / 10$. We carried out solving Eq. (22) starting from a small value of $m$. The scaling is terminated when the position of the occurrence of the maximum yield of the nonadiabatic transition does not significantly change for a further increase of $m$.
Now we attempt to analytically extract an optimal pulse duration from Eq. (34a). We approximate the Bessel functions of fractional order $K_{v}(z)$ ( $v=3 / 4$ and 1/4) in Eq. (34a) using its asymptotic form for large arguments,

$$
K_{v}(z) \sim \sqrt{\frac{\pi}{2 z}} e^{-z}\left[1+\frac{4 v^{2}-1}{8 z}\right] .
$$

This approximation becomes valid for $T \rightarrow 0$ and $T \rightarrow \infty$ since the argument $\xi$, given by Eq. (34b), diverges for these limits. The yield of the nonadiabatic ionization then becomes

$$
P_{0} \sim \frac{c_{1} \sqrt{\pi a} \alpha_{0}^{4}}{16 \sqrt{I_{p}}} \frac{\left(I_{p} T\right)^{2}}{\left[\left(I_{p} T\right)^{2}+c_{2}\right]^{\frac{3}{2}}} e^{-\frac{\left(I_{p} T\right)^{2}}{4 a}} .
$$

The position of maximum is found solving $d P_{0} / d\left(I_{p} T\right)=0$. Then we obtain

$$
\left(I_{p} T\right)^{4}+\left(2 a+c_{2}\right)\left(I_{p} T\right)^{2}-4 a c_{2}=0 .
$$

The solution is

$$
I_{p} T=\sqrt{\frac{1}{2}\left[-2 a-c_{2}+\sqrt{4 a^{2}+20 a c_{2}+c_{2}^{2}}\right] .}
$$

We first demonstrate the result given by Eq. (38b) for a model potential of $\mathrm{H}^{-}$given by Eq. (29). The parameters $c_{1}$ and $c_{2}$ for the ansatz given by Eq. (33) are found using fitting procedures; we obtain $c_{1}=1.76 \times 10^{-3}$ and $c_{2}=0.301$. The

TABLE I. The different combination of the parameters $W_{0}$ and $\sigma$ for the atomic potential given by Eq. (39) so that a field-free ground-state energy becomes $E_{0}( \pm \infty)=-0.1$. The parameters $c_{1}$ and $c_{2}$ are used for the ansatz [Eq. (33)] to fit the matrix element $D(k)$ [Eq. (30b)]. The value of $T_{\text {asym }}$ is a predicted peak position of the detachment yield by the nonadiabatic transition [Eq. (34a)] using the asymptotic formula given by Eq. (38b).

\begin{tabular}{lccccc}
\hline \hline & $W_{0}$ & $\sigma$ & $c_{1}$ & $c_{2}$ & $T_{\text {asym }}$ \\
\hline Case I & 0.345 & 1 & 0.119 & 0.155 & 5.19 \\
Case II & 0.159 & 4 & 0.00641 & 2.73 & 14.2 \\
\hline \hline
\end{tabular}



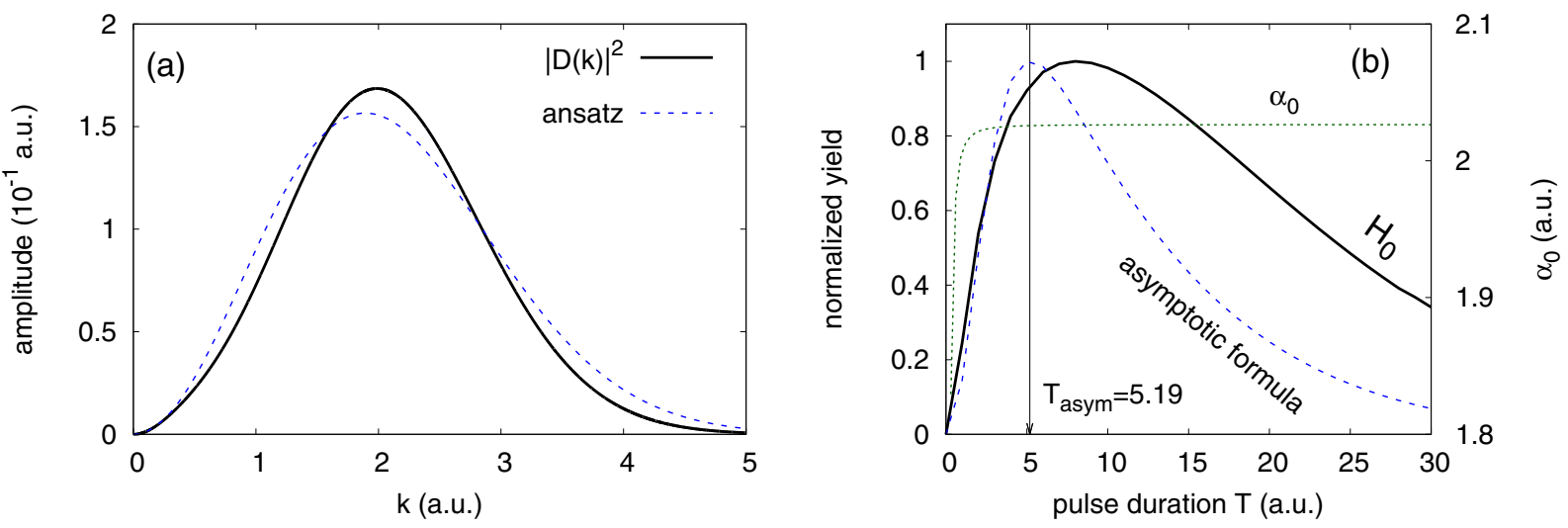

FIG. 4. The same as Fig. 3, but for another atomic potential [Eq. (39)] with depth $W_{0}$ and the width $\sigma$ referred to as case I in Table I.

result of the fitting is shown in Fig. 3(a). The solid (black) and dashed (blue) lines show the results obtained by Eqs. (30b) and (33), respectively. We find that both of the results agree very well. Figure 3(b) shows the detachment yield of the nonadiabatic transition. The solid (black) and dashed (blue) line are obtained by the TDSE given by Eq. (22) and the asymptotic formula given by Eq. (37), respectively. The heights of these lines are normalized to unity to clearly compare the peak positions. The scaling procedure given by Eq. (35) is terminated at $m=8$. Substituting the value of the ionization potential $I_{p}=0.0277$ and $c_{2}=0.301$ to Eq. (38b), the expected optimal pulse duration is found to be $T_{\text {asym }}=24.8$. This is about $20 \%$ off the exact value $T=31$ shown in Fig. 3. We consider that this is reasonable in the rough approximations.

\section{Effect of atomic structure on optimal pulse duration}

We find that Eq. (38b) depends on the constant $c_{2}$, which is related to the curvature of the atomic potential $V(x)$. In the harmonic approximation of the atomic potential, the curvature gives us a ground-state energy; see Appendix B. To see the effects of the atomic structure on the optimal pulse duration, let us consider another atomic potential,

$$
W(x)=-W_{0} e^{-(x / \sigma)^{2}},
$$

with the different combinations of the parameters $W_{0}$ and $\sigma$ summarized in Table I, which are referred to as cases I and II, respectively. Case I (II) represents a deep and narrow (shallow and wide) atomic potential. These combinations are chosen so that the field-free ground-state energy becomes $E_{0}( \pm \infty)=$ -0.1 . The values of $c_{1}$ and $c_{2}$ for the ansatz given by Eq. (33) are summarized in Table I. We repeated the scaling procedure given by Eq. (35) to reach the high-frequency limit $\omega \rightarrow \infty$. In case I (II), the peak position of the maximum yield of the nonadiabatic transition is converged for $m=32(m=16)$. Substituting the parameter $c_{2}$ into Eq. (38b), which is given in Table I, we obtain

$$
\begin{aligned}
& \text { case I : } I_{p} T \approx 0.519 \rightarrow T_{\text {asym }}=5.19, \\
& \text { case II }: I_{p} T \approx 1.42 \rightarrow T_{\text {asym }}=14.2 .
\end{aligned}
$$

These values are also found in Table I. Results are shown in Figs. 4 and 5 for cases I and II, respectively, in the manner of Fig. 3. The quality of the asymptotic expansion of the modified Bessel function given by Eq. (36) near the origin for case II is better than case I due to the bigger value of $c_{2}$; see Table I. So, we obtain the better result for the position of the maximum yield in case II than case I.

It was shown in Fig. 4 of [16] that the yield of the nonadiabatic transition has a maximum for a certain value of a pulse duration, and it was estimated using $I_{p} T \approx 1$, which is equivalent to Eq. (38b) with the right-hand side being unity.
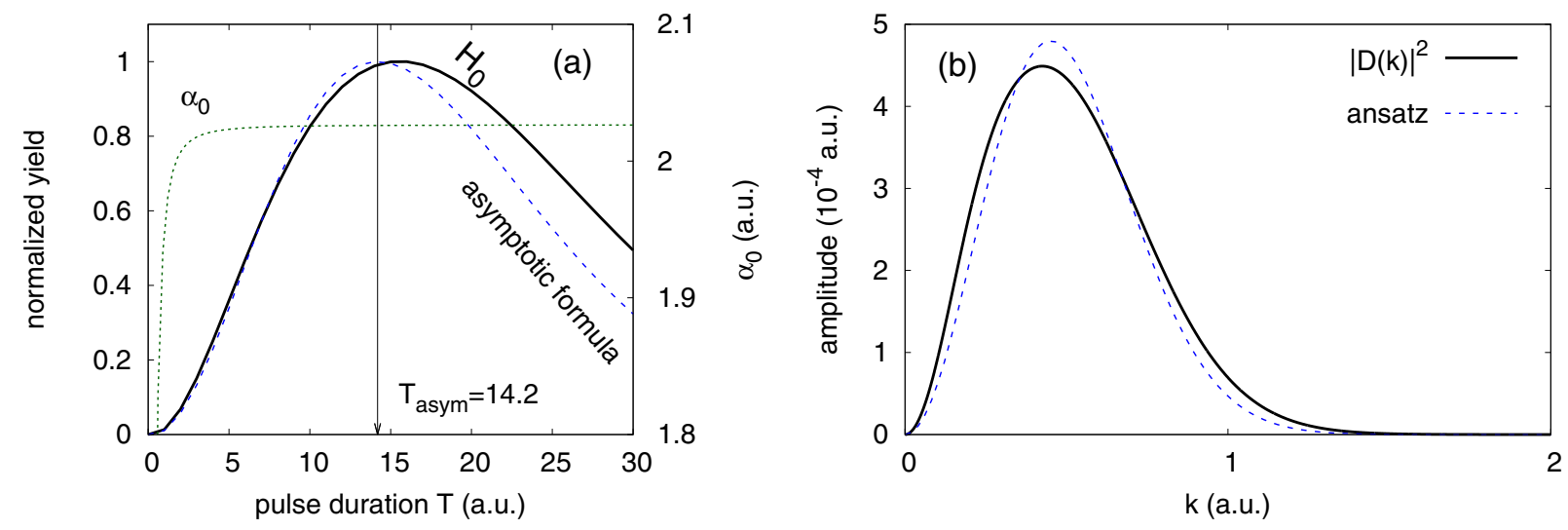

FIG. 5. The same as Fig. 3, but for another atomic potential [Eq. (39)] with depth $W_{0}$ and the width $\sigma$ referred to as case II in Table I. 
However, the right-hand side of Eq. (38b) depends on the curvature of a target potential, as shown in this demonstration.

\section{CONCLUSION}

Following the previous work [6], we further explored a detachment dynamics of a model negative ion in the highfrequency regime. We revisited the interference substructures in photon-absorption peaks in an adiabatic approximation based on the envelope Hamiltonian. The adiabatic approximation clarified that two different interference mechanisms are responsible for its emergence. The first mechanism is the spatial interference. At a certain time in a rising part of a pulse, two photoelectron wave packets are launched at two turning points of a classical electron in the pulse. An interference of them creates a net amount of a photoelectron wave packet. This spatial interference is repeated in the falling part of the pulse. Then these photoelectron wave packets produced in different moments in time cause the temporal interference. We confirmed that the adiabatic approximation can well reproduce the oscillating substructure obtained from the full time-dependent Schrödinger equation (TDSE). The interference substructure was previously found in [7], and recently the same was confirmed for a hydrogen atom in [8] and also for a hydrogen molecular ion in $[9,10]$. We showed that our theory is consistent with these known results. In [7], they predicted the coexistence of the spatial and temporal interference. However, their formulation was based on an empirical approach bringing a quasistatic picture into the high-frequency Floquet theory [2]. Here we find both interference mechanisms, derived from first principles.

We also extracted an optimal pulse duration to maximize a detachment yield by nonadiabatic transition. We clarified that the yield is maximized for a pulse duration close to the time scale of the nonadiabatic transition, roughly estimated by Eq. (38b).

Our demonstrations have been done utilizing short-range potential, although our formulation does not depend on the dimensionality and properties of the atomic potential [6]. Further studies in three dimensions in a real atomic system will be worked out in the future.

\section{ACKNOWLEDGMENTS}

K.T. would like to thank Prof. Ulf Saalmann and Prof. Jan M. Rost at the Max Planck Institute for the Physics of Complex Systems, Dresden.

\section{APPENDIX A: DERIVATION OF EQUATION (34a)}

Substituting Eq. (33) into (32), and integrating over $k$, the ionization yield of the nonadiabatic transition $P_{0}(T)$ is written as

$$
\begin{aligned}
P_{0}(T) & =\int_{-\infty}^{\infty}\left|C_{0 \omega}(k)\right|^{2} \frac{d k}{2 \pi} \\
& =\frac{c_{1} \alpha_{0}^{4} \beta}{4} e^{-\frac{\left(I_{p} T\right)^{2}}{4 a}} \int_{-\infty}^{\infty} k^{2} e^{-\beta k^{4}-\gamma k^{2}} d k,
\end{aligned}
$$

where

$$
\begin{aligned}
& \beta=\frac{T^{2}}{16 a}, \\
& \gamma=\frac{I_{p} T^{2}}{4 a}+\frac{c_{2}}{4 a I_{p}} .
\end{aligned}
$$

The integral can be written using the modified Bessel function $I_{v}(z)$ of the first kind of the fractional order $v$,

$$
\begin{aligned}
& \int_{-\infty}^{\infty} x^{2} e^{-\beta x^{4}-\gamma x^{2}} d x \\
& =\frac{\pi e^{\frac{\gamma^{2}}{8 \beta}}}{8 \sqrt{2} \beta^{\frac{3}{2}} \sqrt{\gamma}}\left[-\gamma^{2} I_{-\frac{1}{4}}\left(\frac{\gamma^{2}}{8 \beta}\right)+\left(4 \beta+\gamma^{2}\right) I_{\frac{1}{4}}\left(\frac{\gamma^{2}}{8 \beta}\right)\right. \\
& \left.\quad+\gamma^{2}\left\{-I_{\frac{3}{4}}\left(\frac{\gamma^{2}}{8 \beta}\right)+I_{\frac{5}{4}}\left(\frac{\gamma^{2}}{8 \beta}\right)\right\}\right] .
\end{aligned}
$$

Using the properties of $I_{v}(z)$ [22],

$$
\begin{gathered}
I_{\nu-1}(z)-I_{\nu+1}(z)=\frac{2 v}{z} I_{\nu}(z), \\
K_{v}(z)=\frac{\pi}{2} \frac{I_{-v}(z)-I_{\nu}(z)}{\sin (\nu \pi)},
\end{gathered}
$$

where $K_{v}(z)$ is the modified Bessel function of the second kind, the integral can be simplified to

$$
\int_{-\infty}^{\infty} x^{2} e^{-\beta x^{4}-\gamma x^{2}} d x=\frac{1}{8}\left(\frac{\gamma}{\beta}\right)^{\frac{3}{2}} e^{\frac{\gamma^{2}}{8 \beta}}\left[K_{\frac{3}{4}}\left(\frac{\gamma^{2}}{8 \beta}\right)-K_{\frac{1}{4}}\left(\frac{\gamma^{2}}{8 \beta}\right)\right] .
$$

Therefore, we obtain

$$
P_{0}(T)=\frac{c_{1}}{2^{\frac{15}{4}} a^{\frac{1}{4}}} \alpha_{0}^{4} \sqrt{T} \xi^{\frac{3}{2}} e^{\xi^{2}-\frac{\left(I_{p} T\right)^{2}}{4 a}}\left[K_{\frac{3}{4}}\left(\xi^{2}\right)-K_{\frac{1}{4}}\left(\xi^{2}\right)\right],
$$

where

$$
\xi=\frac{1}{\sqrt{8 a}} \frac{\left(I_{p} T\right)^{2}+c_{2}}{I_{p} T}
$$

\section{APPENDIX B: PHYSICAL MEANING OF THE CONSTANT $c_{2}$}

The time-independent Schrödinger equation for the ground state $\phi_{0}$ with the energy $E_{0}$ reads

$$
\left[-\frac{1}{2} \frac{d^{2}}{d x^{2}}+V(x)\right] \phi_{0}(x)=E_{0} \phi_{0}(x) .
$$

Let us consider the Taylor expansion of the atomic potential $V(x)$ around the origin up to the order of $x^{2}$,

$$
V(x) \approx V(0)+\frac{1}{2} \Omega^{2} x^{2},
$$

where $\Omega^{2}=V^{\prime \prime}(0)$ represents the second derivative of the atomic potential $V(x)$ at the origin. In this approximation, the ground state $\phi_{0}^{(0)}(x)$ and its energy level $E_{0}^{(0)}$ thus correspond 
to those of a simple harmonic oscillator, which are given by

$$
\begin{aligned}
\phi_{0}^{(0)}(x) & =\left(\frac{\Omega}{\pi}\right)^{\frac{1}{4}} e^{-\frac{\Omega}{2} x^{2}}, \\
E_{0}^{(0)} & =V(0)+\frac{\Omega}{2} .
\end{aligned}
$$

In what follows, we assume the condition of $E_{0} \approx E_{0}^{(0)}<0$. We exclude considering the case of $E_{0}^{(0)}>0$, which can happen for steep atomic potentials.

Now let us calculate the matrix element $D(k)$ given by Eq. (30b). To this end, we consider the case of $k \gg 1$. Then the scattering state can be replaced with $\langle k, t= \pm \infty|=e^{i k x}$. We approximate the ground-state wave function $\left|\phi_{0}(-\infty)\right\rangle$ using Eq. (B3a). With these assumptions, substituting the expansion Eq. (B2) into Eq. (30b),

$$
\begin{aligned}
|D(k)|^{2} & =\left|\left\langle k, t=-\infty\left|V^{\prime \prime}(x)\right| \phi_{0}(-\infty)\right\rangle\right|^{2} \\
& \approx \frac{\Omega^{\frac{9}{2}}}{\pi^{\frac{1}{2}}}\left|\int_{-\infty}^{\infty} e^{-\frac{1}{2} \Omega x^{2}} e^{i k x} d x\right|^{2} \\
& =2 \sqrt{\pi} \Omega^{\frac{7}{2}} e^{-\frac{k^{2}}{\Omega}}
\end{aligned}
$$

This is the asymptotic formula of Eq. (33) for $k \gg 1$. Therefore, we obtain

$$
\frac{c_{2}}{4 a I_{p}}=\frac{1}{\Omega}, \rightarrow c_{2}=\frac{4 a I_{p}}{\Omega} .
$$

For the one-dimensional (1D) model of $\mathrm{H}^{-}$, given by Eq. (29), and case $I$ in Table I for the Gaussian potential, given by Eq. (39), the ground-state energy with harmonic approximation is bigger than 0. So, the formulation in this appendix is not applicable. For case II in Table I, $V^{(2)}(0)=0.02$ and $I_{p}=0.088$. On the other hand, the exact value is $I_{p}=0.1$. Then Eq. (B5) gives us $c_{2}=3.45$, while the exact value shown in Table $\mathrm{I}$ is $c_{2}=2.72$.

\section{APPENDIX C: UNIFORM APPROXIMATION}

The formulation here follows Berry [21]. To implement the uniform approximation, we introduce the mapping for Eq. (17),

$$
\Phi_{n}(t)=-\int_{-\infty}^{t} E_{0}\left(t^{\prime}\right) d t^{\prime}-n \omega t+E t=\zeta y+\frac{y^{3}}{3}+X .
$$

Let $t=t_{ \pm}$satisfy the stationary phase condition,

$$
\left(\frac{d \Phi}{d t}\right)_{t_{ \pm}}=-E_{0}\left(t_{ \pm}\right)-\omega+E=\left(\zeta+y^{2}\right)\left(\frac{d y}{d t}\right)_{t_{ \pm}}=0
$$

then these are mapped onto

$$
y_{ \pm}= \pm i \sqrt{\zeta} .
$$

Realizing $y_{+}=-y_{-}$, the constant $X$ is given by

$$
X=\frac{1}{2}\left[\Phi\left(t_{-}\right)+\Phi\left(t_{+}\right)\right]
$$

Substituting Eqs. (C3) and (C4) into Eq. (C1), we obtain

$$
\zeta=\left[-\frac{3 i}{2} \theta\left(t_{+}\right)\right]^{\frac{2}{3}}
$$

Another mapping we need is

$$
\frac{d t}{d y} M_{n \omega}(k, t)=p+q y .
$$

The function $M_{n \omega}(k, t)(n \neq 0)$ is defined by Eq. (21b). Substituting $y=y_{ \pm}$into this equation, the constants $p$ and $q$ are determined as

$$
\begin{aligned}
& p=\frac{1}{2}\left[\left(\frac{d t}{d y}\right)_{y_{+}}+\left(\frac{d t}{d y}\right)_{y_{-}}\right] M_{n \omega}\left(k, t_{+}\right) \\
& q=-\frac{i}{2 \sqrt{\zeta}}\left[\left(\frac{d t}{d y}\right)_{y_{+}}-\left(\frac{d t}{d y}\right)_{y_{-}}\right] M_{n \omega}\left(k, t_{+}\right)
\end{aligned}
$$

Note that the matrix element of the $n$ photon absorption takes the same value at $t=t_{ \pm}$. To calculate the value of $d t / d y$, we twice differentiate Eq. (C1) by $y$,

$$
2 y=\left[-E_{0}(t)-\omega+E\right] \frac{d^{2} t}{d y^{2}}-\frac{d E_{0}}{d t}\left(\frac{d t}{d y}\right)^{2} .
$$

Realizing that $d t / d y$ is an even function, substituting either $y=y_{-}$or $y_{+}$, we obtain

$$
\left(\frac{d t}{d y}\right)_{y_{ \pm}}=\sqrt{\frac{-2 i \zeta^{1 / 2}}{\dot{E}_{0}\left(t_{+}\right)}}
$$

or

$$
\left(\frac{d t}{d y}\right)_{y_{ \pm}}=-\sqrt{\frac{-2 i \zeta^{1 / 2}}{\dot{E}_{0}\left(t_{+}\right)}}
$$

Substituting this into Eq. (C7), we thus obtain

$$
\begin{aligned}
& p= \pm \sqrt{\frac{-2 i \zeta^{1 / 2}}{\dot{E}_{0}\left(t_{+}\right)}} M_{n \omega}\left(k, t_{+}\right), \\
& q=0 .
\end{aligned}
$$

The positive and negative sign of $p$ corresponds to the solution given by Eq. (C9a) or (C9b), respectively. Therefore, the photoelectron amplitude for single-photon absorption, given by Eq. (21a) for $n=1$, is given by

$$
\begin{aligned}
C_{n \omega}^{(1)}(k) & =e^{i X} \int_{-\infty}^{\infty} \frac{d t}{d y} M_{n \omega}(k, t) e^{i\left(\zeta y+\frac{y^{3}}{3}\right)} d y \\
& = \pm 2 \pi e^{i X} \sqrt{\frac{-2 i \zeta^{1 / 2}}{E_{0}^{\prime}\left(t_{+}\right)}} M_{n \omega}\left(k, t_{+}\right) \operatorname{Ai}(\zeta) \\
& = \pm e^{i X} \sqrt{\frac{-4 \theta\left(t_{+}\right)}{\dot{E}_{0}\left(t_{+}\right)}} M_{n \omega}\left(k, t_{+}\right) J_{\frac{1}{3}}\left[-\theta\left(t_{+}\right)\right] .
\end{aligned}
$$


The function $\operatorname{Ai}(z)$ represents the Airy function. Here we used, on the last line [22],

$$
\begin{aligned}
\operatorname{Ai}(z) & =\frac{1}{\pi} \sqrt{\frac{z}{3}} K_{\frac{1}{3}}\left(\frac{2}{3} z^{\frac{3}{2}}\right), \\
K_{v}(z) & =J_{v}(i z)
\end{aligned}
$$

where the function $K_{v}(z)$ and $J_{v}(z)$ are the modified Bessel function of fractional order $v$ and the Bessel function of fractional order $v$, respectively. Substituting the asymptotic form of the matrix element for the $n$ photon absorption given by Eq. (25a) into Eq. (C11),

$$
\begin{aligned}
C_{n \omega}^{(1)}(k)= & \pm i^{n} e^{i(X-n \delta)} \sqrt{\frac{4 \theta\left(t_{+}\right)}{\dot{E}_{0}\left(t_{+}\right)}} A\left(k\left(t_{+}\right)\right) \varphi_{0}\left(-\alpha\left(t_{+}\right)\right) \\
& \times J_{n}\left(\left|k\left(t_{+}\right)\right| \alpha\left(t_{+}\right)\right) J_{\frac{1}{3}}\left(-\theta\left(t_{+}\right)\right) .
\end{aligned}
$$

It is found that the spectrum is written using two Bessel functions. The Bessel function of the integer order $n$ represents the spatial interference, and the fractional order $1 / 3$ represents the temporal interference. It is easily shown by l'Hôpital's rule that the quantity $\theta\left(t_{+}\right) / \dot{E}_{0}(t)$ is of the order of $t_{+}^{2}$ at the vicinity of $t_{+}=0$. Therefore, the result given by Eq. (C13) does not have the singularity at $t_{+}=0$.
[1] M. Chini, K. Ahao, and Z. Chang, Nat. Photon. 8, 178 (2014).

[2] M. Gavrila and J. Z. Kaminski, Phys. Rev. Lett. 52, 613 (1984).

[3] W. C. Henneberger, Phys. Rev. Lett. 21, 838 (1968).

[4] M. Pont and M. Gavrila, Phys. Rev. Lett. 65, 2362 (1990).

[5] L. You, J. Mostowski, and J. Cooper, Phys. Rev. A 45, 3203 (1992).

[6] K. Toyota, U. Saalmann, and J. M. Rost, New J. Phys. 17, 073005 (2015).

[7] K. Toyota, O. I. Tolstikhin, T. Morishita, and S. Watanabe, Phys. Rev. A 76, 043418 (2007).

[8] P. V. Demekhin and L. S. Cederbaum, Phys. Rev. Lett. 108, 253001 (2012).

[9] C. Yu, N. Fu, T. Hu, G. Zhang, and J. Yao, Phys. Rev. A 88, 043408 (2013).

[10] L. Yue and L. B. Madsen, Phys. Rev. A 90, 063408 (2014).

[11] R. R. Jones, Phys. Rev. Lett. 74, 1091 (1995).

[12] V. C. Reed and K. Burnett, Phys. Rev. A 43, 6217 (1991).
[13] D. Barash, A. E. Orel, and R. Baer, Phys. Rev. A 61, 013402 (1999).

[14] M. Førre, S. Selstø, J. P. Hansen, and L. B. Madsen, Phys. Rev. Lett. 95, 043601 (2005).

[15] K. Toyota, O. I. Tolstikhin, T. Morishita, and S. Watanabe, Phys. Rev. Lett. 103, 153003 (2009).

[16] O. I. Tolstikhin, Phys. Rev. A 77, 032711 (2008).

[17] M. Tilly, A. Karamatskou, and R. Santra, J. Phys. B 48, 124001 (2015).

[18] M. Pont, N. R. Walet, M. Gavrila, and C. W. McCurdy, Phys. Rev. Lett. 61, 939 (1988).

[19] M. Pont, Phys. Rev. A 44, 2141 (1991).

[20] A. M. Popov, O. V. Tikhonova, and E. A. Volkova, J. Phys. B: At. Mol. Opt. Phys. 32, 3331 (1999).

[21] M. V. Berry, Proc. Phys. Soc. 89, 479 (1966).

[22] F. W. J. Olver, D. W. Lozier, R. F. Boisvert, and C. W. Clark, NIST Handbook of Mathematical Functions (Cambridge University Press, New York, 2010). 